\title{
Diseño de un robot rescatista para terremotos en México
}

\author{
Jesús Álvarez Cedillo ${ }^{1}$, Teodoro Álvarez Sánchez ${ }^{2}$, Raúl Junior Sandoval ${ }^{1}$, \\ Jacobo Sandoval Gutiérrez ${ }^{3}$, Adriana Nava Vega ${ }^{4}$ \\ ${ }^{1}$ Instituto Politécnico Nacional, UPIICSA, Ciudad de México, México \\ jalvarez@ipn.mx \\ ${ }^{2}$ Instituto Politécnico Nacional, CITEDI, Baja California, México \\ talvarez@citedi.mx \\ ${ }^{3}$ Universidad Autónoma Metropolitana, Unidad LERMA, Estado México, México \\ jacobosandovalg@gmail.com \\ ${ }^{4}$ Universidad Autónoma de Baja California, Campus Tijuana, México \\ adriana.nava@uabc.edu.mx
}

Resumen. La República Mexicana está situada en una de las regiones sísmicamente más activas del mundo, enclavada dentro del área conocida como el Cinturón Circumpacífico donde se concentra la mayor actividad sísmica del planeta. En los últimos 5 años se han producido terremotos de magnitud muy considerable afectando estados como Oaxaca, Puebla, Morelos, Guerrero, Michoacán y la Ciudad de México. El número de personas que murieron atrapadas dentro de los escombros fue muy alta y en los próximos años se seguirá teniendo actividad sísmica constante. La necesidad de desarrollar un robot que permita detectar personas atrapadas no como un prototipo si no como una herramienta profesional de rescate es una prioridad. En este trabajo se propone el desarrollo de un robot rescatista diseñado con diversos sensores para detectar la temperatura, ruidos, detectar respiración de personas en los escombros. El robot propuesto se moverá entre los desechos para detectar a los humano.

Palabras clave: robótica, aplicaciones de robótica, algoritmos de navegación, robot rescatista.

\section{Design of a Rescue Robot for Earthquakes in Mexico}

\begin{abstract}
The Mexican Republic is located in one of the most seismically active regions in the world, nestled within the area known as the Circumpacific Belt where the largest seismic activity on the planet is concentrated. In the last 5 years, there have been earthquakes of very considerable magnitude affecting states such as Oaxaca, Puebla, Morelos, Guerrero, Michoacán and Mexico City. The number of people who died trapped inside the rubble was very high and in the coming years, there will continue to be constant seismic activity. The need to develop a robot that allows detecting people trapped not as a prototype but as a professional rescue tool is a priority. This work proposes the development of a rescue robot designed with various sensors to detect temperature, noise, detect breathing of people in the rubble. The proposed robot will move through the waste to detect humans.
\end{abstract}

Keywords: robotics, robotics applications, navigation algorithms, rescue robot. 


\section{Introducción}

En las últimas dos décadas se estima que los desastres son responsables de aproximadamente 3 millones de muertes en todo el mundo, 800 millones de personas afectadas adversamente y daños a la propiedad que superan los 50 mil millones de dólares. El reciente terremoto en México del 19 de septiembre de 2017 dejo una gran cantidad de personas muertas y heridas. Muchas de estas muertes se debieron a un colapso estructural cuando los edificios cayeron sobre las personas. La Búsqueda y Rescate Urbano involucra la ubicación, el rescate (extracción) y la estabilización médica inicial de las víctimas atrapadas en espacios confinados. Los vacíos que se forman cuando un edificio colapsa es una instancia de un espacio confinado. La Búsqueda y Rescate Urbano puede ser necesaria para una variedad de situaciones, incluyendo terremotos, huracanes, tornados, incendios, actividades terroristas $\mathrm{y}$ accidentes con materiales peligrosos.

En la actualidad, un equipo típico de búsqueda y rescate está compuesto por una decena de personas, entre las que se incluyen perros y adiestradores caninos, un paramédico, un ingeniero de estructuras y varios especialistas en el manejo de equipo especial para encontrar y extraer a una víctima. El equipo de búsqueda actual incluye cámaras de búsqueda y dispositivos de escucha. Las cámaras de búsqueda suelen ser cámaras de video montadas en algún dispositivo, como un poste que se puede insertar en huecos y agujeros para buscar signos de personas. A menudo, se perfora un agujero en las paredes obstruidas si se sospecha que existe un vacío en el otro lado. También se utiliza la imagen térmica. Esto es especialmente útil para encontrar cuerpos cálidos que han sido recubiertos con polvo y escombros que camuflajean efectivamente a la víctima. Los dispositivos utilizados que detectan sonidos son micrófonos altamente sensibles que pueden escuchar a una persona que puede estar moviéndose o intentando responder a las llamadas de los rescatistas.

Un temblor, sismo o terremoto, consiste en una serie de vibraciones en las rocas que se propagan en todas direcciones en forma de ondas. Estas ondas sísmicas son producidas por la liberación de energía ocurrida en los movimientos de grandes bloques de roca, los que son generados en grandes fallas (límites de placas tectónicas en separación, en colisión o en deslizamiento lateral); existen también temblores menores o locales (producidos por fallas pequeñas y por explosiones provocadas por volcanes o por el hombre) [1].

La voz popular denomina temblor a aquellos movimientos que son sentidos por el hombre (baja magnitud); sismo, al temblor que se siente y que causa algunos destrozos en construcciones (mediana magnitud); terremoto, a aquellos movimientos que son tan fuertes que producen graves daños al hábitat del hombre (gran magnitud).

La "Sismología" estudia los temblores, sismos o terremotos y sus ondas sísmicas, registradas en forma precisa por instrumentos denominados sismógrafos.

La amplitud de las ondas sísmicas permite clasificar a los temblores dentro de una escala denominada "Escala de Richter" (de uso mundial). Su magnitud está basada en el logaritmo de la amplitud máxima de la onda sísmica, ajustada por un factor que toma en cuenta la intensidad o debilidad de las ondas y su dispersión a partir del foco (centro de dispersión de las vibraciones de un temblor). Debido a que estas magnitudes están basadas en una escala logarítmica, un incremento en magnitud de una unidad, corresponde a un incremento de 10 veces, en el tamaño de un temblor, 
por ejemplo un temblor de magnitud 8 será 10,000 veces mayor que un temblor de magnitud 4 (esto es 108/104).

Cada año ocurren numerosos temblores registrados por sismógrafos, pequeños de magnitud inferior a 1 hasta magnitud 3.4, sin ser sentidos por el humano; a partir de la magnitud 5 empiezan a ocurrir daños y son sentidos prácticamente por todos, incrementándose estos daños en temblores de intensidad superior hasta llegar a 8 y más, con la casi destrucción total, siendo estos grandes temblores, que ocurren cada 5 a 10 años, registrados en la historia por los grandes disturbios provocados.

Debido a la gran sismicidad reportada de sismos en México, nuestro proyecto de investigación consiste en diseñar, construir y programar un robot autónomo capaz de búsqueda de víctimas en un edificio colapsado por el terremoto y justificar un robot no prototipo.

Los puntos a destacar que deben de ser cubiertos es que debe de desplazarse en un cuadrado de prueba de $20 \times 20$ metros., inclinaciones de hasta 50 grados, debe de evitar obstáculos y detectar sobrevivientes.

Es posible contar con un mapa de la construcción, pero los obstáculos resultantes del terremoto se dejaron como incógnitas. Nuestro robot tiene que acercarse a las víctimas identificadas y reportar a la central. Un sensor GPS muestra la posición real del robot en el espacio.

Los desastres naturales recientes y las catástrofes provocadas por el hombre han centrado la atención en el área de manejo de emergencias y rescate. Estas experiencias han demostrado que la mayoría de las respuestas de preparación y emergencia del gobierno son generalmente inadecuadas para hacer frente a los desastres. Teniendo en cuenta la gran cantidad de personas que han muerto debido a esfuerzos de rescate reactivos, espontáneos y no profesionales como resultado de la falta de equipo adecuado o la falta de respuesta inmediata, los investigadores naturalmente han desarrollado herramientas de rescate mecatrónicas y técnicas de planificación estratégica para las operaciones de rescate planificadas. Las actividades de investigación y desarrollo han dado lugar al surgimiento del campo de la robótica de rescate, que puede definirse como la utilización de tecnología robótica para la asistencia humana en cualquier fase de las operaciones de rescate, que son multifacéticas y varían de un país a otro debido a las políticas regionales. Los tipos de desastres y las diferentes composiciones de escombros en las áreas de desastre. Otros aspectos de la robótica de rescate incluyen:

1. Detección e identificación de cuerpos vivos.

2. Enrutamiento y / o limpieza de escombros para acceder a la víctima.

3. Estabilización física, emocional o médica del sobreviviente al proporcionarle primeros auxilios telemétricos y de administración automática.

4. Fortificación del cuerpo vivo para una recuperación segura contra cualquier escombro que caiga y posibles lesiones.

5. Transporte de la víctima.

Estas operaciones también varían en carácter para diferentes tipos de entornos de desastre, como áreas urbanas, subterráneas o bajo el agua, que son desestructuradas y tecnológicamente difíciles de acceder para los humanos. Los problemas críticos en el rescate son la conveniencia y el cumplimiento de las herramientas de rescate. Los otros problemas importantes de rescate encontrados son: 
1. Las herramientas no ásperas son generalmente engorrosas, destructivas y, por lo general, se adaptan directamente a los dispositivos de construcción.

2. Las máquinas de limpieza de escombros son dispositivos de construcción pesada que, cuando funcionan sobre escombros, hacen que los escombros se hundan.

3. El funcionamiento de la herramienta es generalmente muy lento y tedioso y no tiene en cuenta los intentos anteriores en el mismo lugar (no aprenden de las pruebas en el lugar), lo que produce muchas repeticiones fallidas.

4. Aunque hay algunos detectores disponibles, la búsqueda de sobrevivientes se basa principalmente en la detección de perros y voces humanas, donde la llamada y la escucha requieren silencio y atención enfocada que es muy difícil debido a los trabajadores de rescate sobrecargados, agotados y deprimidos.

5. El suministro de primeros auxilios solo se puede hacer cuando se encuentra cerca del sobreviviente, una distancia que se alcanza con frecuencia cuando se excede el momento crítico para la supervivencia.

6. La recuperación de cuerpos genera lesiones adicionales, ya que la estabilización profesional de la víctima rara vez se obtiene y no se supervisa continuamente.

Con el objetivo de mejorar la calidad del rescate y la vida después del rescate, el campo de la robótica de rescate busca dispositivos diestros que estén equipados con capacidad de aprendizaje, adaptables a diversos tipos de uso con una funcionalidad lo suficientemente amplia bajo múltiples sensores y que cumplan con las condiciones del el medio ambiente y el de la persona rescatada.

\section{Descripción del método}

Se plantea la necesidad de desarrollar una plataforma robótica que permita acceder y permanecer en entornos naturales, desplazarse sin inconvenientes por los múltiples terrenos [2].

Nuestro método de construcción se basa en dos áreas fundamentales:

1. El cuerpo del robot: Se decidió que para que este tipo de robot tenga éxito, debe ser capaz de viajar en un camino no muy recto, alcanzar alturas de 20 centímetros, y ser capaz de escalar hasta 60 grados. El diseño debe de mantener su integridad estructural resistente. El robot será capaz de viajar en rampas para comprobar si hay víctimas en diferentes niveles. Por último, las herramientas utilizadas se diseñan de manera modular para adaptación de piezas adicionales o modificaciones del diseño.

2. La exploración: Para llevar a cabo los viajes camino recto y giros precisos se creó un sistema de doble accionamiento diferencial. La ventaja de este tipo de sistema es que un motor se utiliza para el movimiento hacia adelante y hacia atrás, mientras que un segundo motor se utiliza para hacer el escalamiento de obstáculos. Esta configuración tiene la intención de hacer que las dos ruedas motrices se mueven exactamente lo mismo si se viaja hacia delante o hacia atrás o exactamente lo contrario si se activa el motor de escalada. Para probar el éxito de nuestra unidad de doble diferencial que 
mide la forma recta el robot de conducir sin ningún control de retroalimentación y la precisión con la que sería girar 90 grados. Los resultados para la línea recta eran buenas, pero las vueltas tienen un poco de problema.

\subsection{Características mínimas del diseño}

Los parámetros usados en el diseño son los siguientes:

1. Capacidad de desplazamiento en lugares de difícil acceso.

2. Posibilidad de desplazamiento en múltiples tipos de terrenos.

3. Movimiento del chasis con el propósito de poder cambiar la posición de este y adaptarlo a las necesidades mediante el control remoto.

4. Utilizar el control remoto para dirigir el robot a los lugares no accesibles para obtener información visual.

5. Capacidad de visión del robot para obtener acercamientos claros de la zona.

6. Consideraciones para realizar cálculos.

\section{Mecánica del robot}

Los robots de rescate de hoy en día no tienen grandes sistemas de cómputo muy sofisticados en términos de inteligencia artificial (en inglés, AI). La IA es una forma de programar a la computadora que le permite al robot procesar información y tomar algunas decisiones por sí mismo. En lugar de una IA independiente, la mayoría de los robots rescatistas son controlados remotamente por los operadores humanos. Vehículos terrestres no tripulados (UGV) o vehículos aéreos no tripulados (UAV) [3].

Los motores se escogieron en base al cálculo de los momentos críticos en lo que estos se someterían en las peores condiciones y con base a estos cálculos se da un factor de seguridad adecuado en el que se garantice un correcto funcionamiento, igualmente para escoger el material y las dimensiones adecuadas para los ejes se procede a hacer un análisis teniendo en cuenta los esfuerzos cortantes y los momentos vectores en condiciones críticas, entonces se procede a calcular dichas dimensiones y dando un respectivo factor de seguridad para estos, para escoger la relación correcta de engranes para las orugas, se tuvo en cuenta que las velocidades de las orugas y las llantas serán iguales [4].

Como una solución al problema de la plataforma mecánica se planteó inicialmente una plataforma robot explorador terrestre tipo rover de 6 ruedas con sistema de amortiguamiento Rocker bogie, el cual le permite superar obstáculos con facilidad además de garantizar la tracción por lo menos de alguna de las ruedas, acoplándose al terreno, y por lo tanto teniendo ventajas ante sistemas clásicos de todo terreno como la oruga simple. Este sistema permite que la plataforma pueda desplazarse en terrenos donde hay variedad de obstáculos, además de evitar transmisión directa de la energía al chasis debido a este sistema.

Para verificar este sistema se procedió a la construcción de un modelo funcional a escala. La construcción del modelo de menor escala se estableció con el objetivo primordial de analizar la cinemática y la dinámica del sistema mecánico diseñado y 
para realizar las pruebas de los sensores necesarios para el funcionamiento de la plataforma antes de la construcción del prototipo final.

El diseño del modelo a escala se basó en la suspensión Rocker bogie utilizada en varios de los robots construidos por la NASA [5], pero usando el eslabón libre como la parte frontal de la plataforma de esta manera permite sobrepasar obstáculos de mayor dimensión, esto se observó en el prototipo a escala. El diseño del modelo se muestra en la Fig. 1.

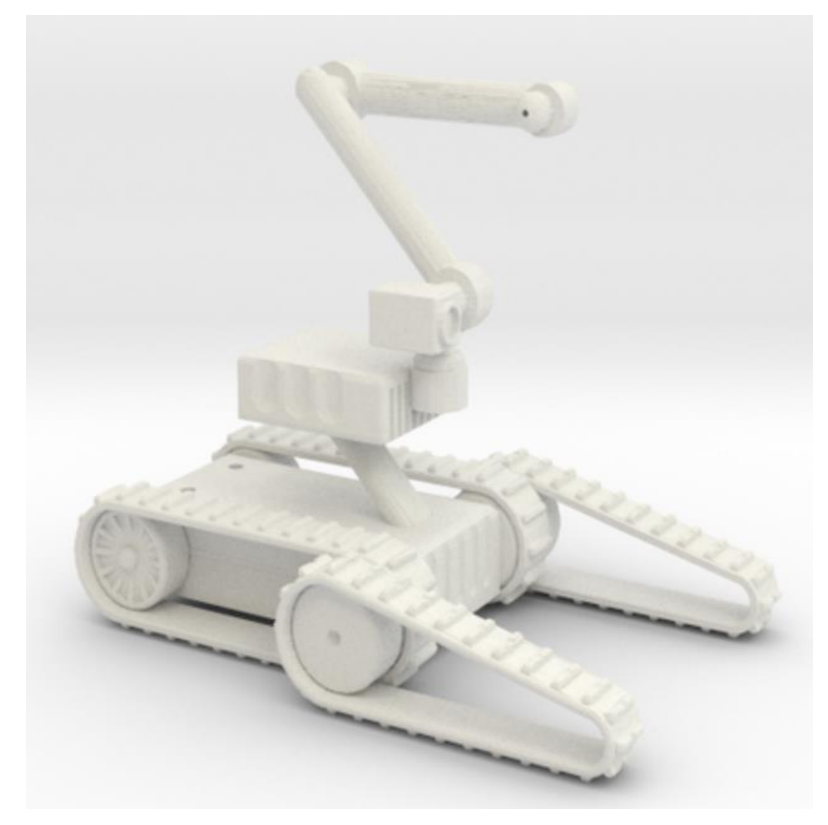

Fig. 1. Prototipo del robot construido.

Los robots más comunes son robots pequeños y planos montados en huellas de tanques en miniatura. Estos robots son resistentes, capaces de ingresar por huecos y edificios y generalmente tienen una variedad de sensores integrados, que incluyen la vigilancia de audio y video y la detección de vida humana. Estos robots son versátiles, con diferentes sensores o herramientas que se pueden montar. Prácticamente todos son portátiles. El robot propuesto se maneja con un joystick de control, tiene siete configuraciones de velocidad (la velocidad máxima es de 1.8 metros por segundo y puede usar sus huellas para subir escaleras, maniobrar entre los escombros e incluso tomar muestras.

Nuestra versión más simple incluye dispositivos de audio y video y un brazo mecánico. Una versión ligera de $27 \mathrm{~kg}$ que omite el brazo.

El robot es controlado por un procesador Pentium con dispositivo empaquetado, un sistema GPS, una brújula electrónica y sensores de temperatura integrados. Puede moverse a más de $8 \mathrm{mph}(13 \mathrm{kph})$, puede desplegarse en minutos y puede soportar una caída de 1,8 metros sobre el concreto, el equivalente a $400 \mathrm{~g}$ de resistencia.

Para su fabricación se realizó el modelo en 3D usando el programa Blender para su diseño. La Fig. 2. Muestra diferentes vistas del modelo en 3D. 

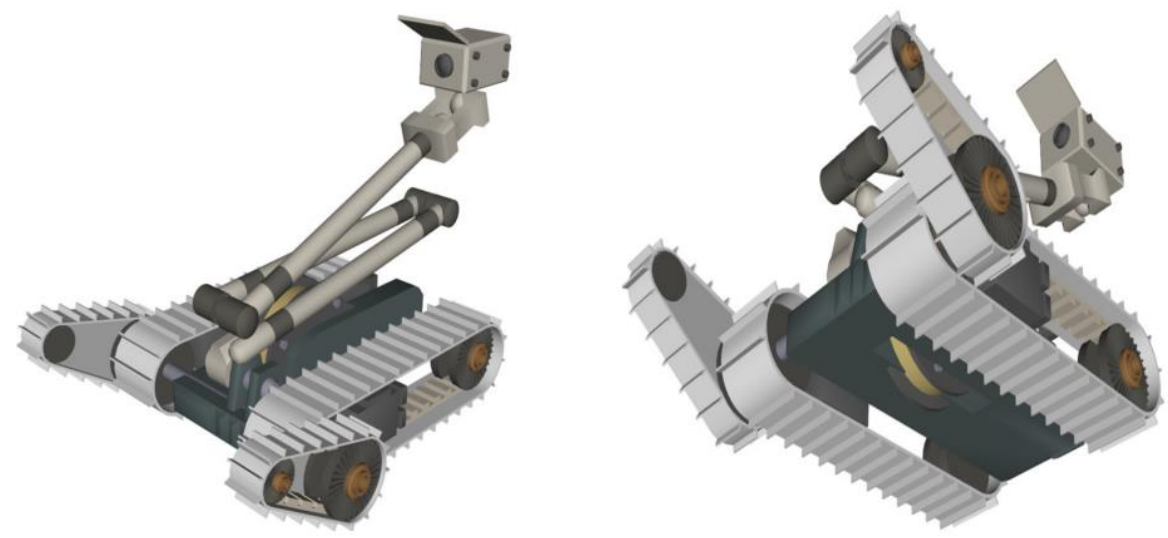

Fig. 2. Vistas del Modelo 3D.

A partir del modelo en 3D creado se maquino cada pieza en aluminio en el Laboratorio de pesados de UPIICSA para tener la estructura y se adquirieron las orugas para el montaje de cada rueda.

El sistema final cuenta de tres motores de 12 volts para accionar las orugas una batería de acido plomo basado en un electrolito de ácido sulfúrico disuelto en agua, 60-110 Wh/l, eficiencia carga/descarga: 50-95\% y temperatura de carga: mín. $-40^{\circ} \mathrm{C}$, máx. $50^{\circ} \mathrm{C}$.

El sistema tiene dos puentes $\mathrm{H}$ para controlar los motores y un sistema basado en Intel para su control y operación.

Otra de las diferencias de la suspensión Rocker bogie original es que los ejes que soportan el eslabón principal no se encuentran conectados mediante una relación diferencial sino que cada uno dispone de un servomotor que controla su movimiento independientemente [5, 6, 9]. El sistema de transmisión del servomotor al eje se realiza mediante engranajes como se puede ver en la Fig. 3.

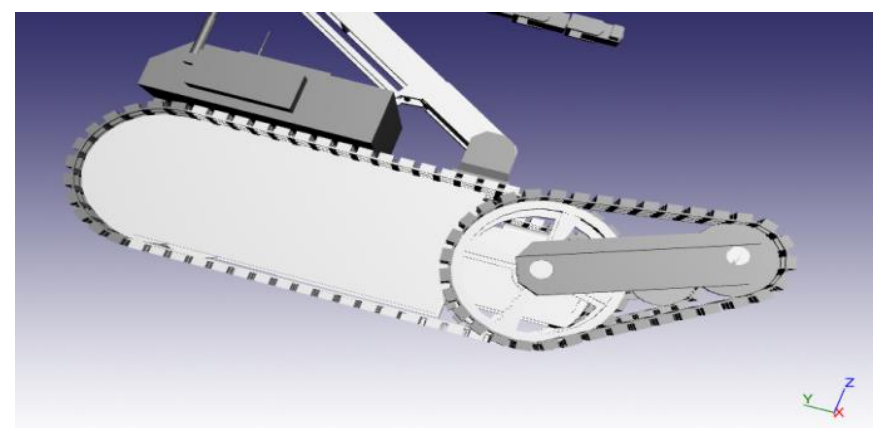

Fig. 3. Sistema de engranajes.

Basados en las ventajas y desventajas halladas mediante las pruebas del modelo a escala se diseño el prototipo a escala real. Se mantiene el acople del armazón al sistema de tracción por medio de un eje a cada lado, ya que esto facilita la absorción 
de momentos, además de contribuir al control del posicionamiento del chasis. Debe tenerse en cuenta que para que el sistema de tracción haga que el robot se desplace debe vencer una fuerza determinada por la ecuación (1), donde $\mu$ corresponde al coeficiente de rodadura entre las ODS superficies en contacto, $\mathrm{m}$ la masa del robot, $\mathrm{g}$ la aceleración debido a la gravedad y $\theta$ el ángulo de la pendiente del suelo con respecto a la horizontal [7]:

$$
r=\mu(m g \cos (\theta))+m g \sin (\theta)+\text { Fin }
$$

El primer término de la derecha de la ecuación 1 (Fr) se refiere a la fuerza de fricción entre las llantas y el suelo que se ve afectado por el ángulo de la pendiente por donde se desplaza. El segundo término se refiere a la componente del peso del robot que al entrar en una pendiente se vuelve significativo. El último término corresponde a las fuerzas inerciales debidas a las aceleraciones causadas por le movimiento del robot. El torque que tendría que hacer cada rueda para mover el robot estaría determinado por la ecuación (2), donde $\mathrm{N}$ corresponde al número de ruedas a tracción [8]:

$$
\begin{gathered}
T r=F r \times \frac{R_{\text {rrueda }}}{N}, \\
T m=f(m g) . R_{-} \text {rueda } .
\end{gathered}
$$

El torque que se le puede pedir a una rueda está determinado por el peso del robot y el coeficiente de fricción f entre el suelo y las llantas según la ecuación (3).

De esta forma para que las ruedas transmitan el torque requerido sin deslizar, este torque debe ser menor al torque máximo tractivo $(\mathrm{Tm})$. Con respecto a las ruedas delanteras, uno de los inconvenientes que se encontró fue la falta de una rueda que completara un triangulo en el eslabón libre de la suspensión Rocker bogie para que cuando este eslabón girara completamente al tratar de sobrepasar obstáculos de tamaño superior al modelo, el robot pueda seguir funcionando correctamente. Para esto se utilizó un sistema de reducción Epicicloide. Para superar los percances detectados se diseñó un prototipo a escala real. El prototipo se muestra en la Fig. 4, donde se aprecian cambios respecto a la versión anterior.

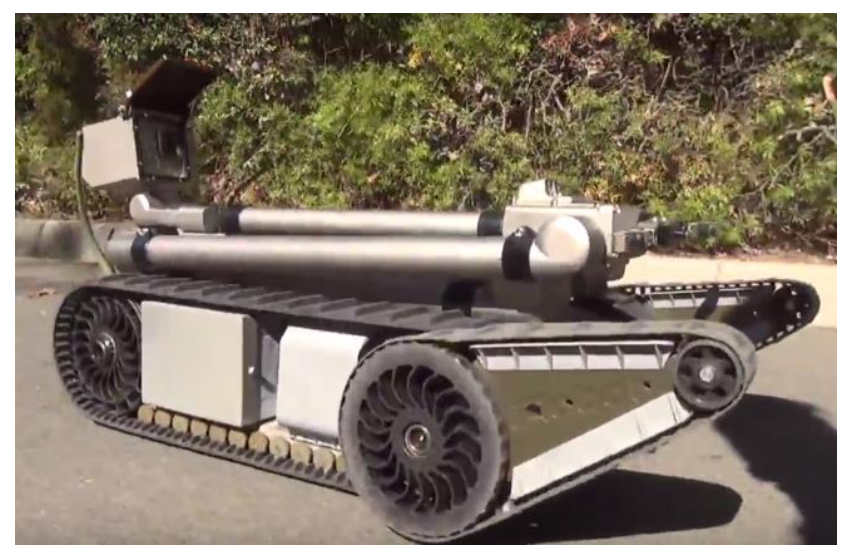

Fig. 4. Robot final. 


\section{Sistema de control remoto}

Tres son los objetivos fundamentales de la estación. El primer objetivo es simplificar la tarea del operador para ello el sistema contará con:

1) Interface hombre/ maquina: Nuestro sistema fue desarrollado con LIVE CODE community.

2) Visualizar la información que proviene de las cámaras panorámicas del robot.

3) Mostrar los datos provenientes de la cámara del brazo manipulador, así como los datos de los sensores.

4) Mostrar los datos de los sistemas de posicionamiento global e inercial, así como la posición actual del manipulador.

5) Proporcionar una herramienta de manipulación (joystick) que permitan manipular tanto el robot como el manipulador de una manera intuitiva.

\section{Resultados}

Al haber realizado la plataforma robot rescatador para terremotos y realizar las pruebas pertinentes, se obtienen los diferentes resultados los cuales se encuentran descritos a continuación:

En la modificación realizada al sistema de suspensión Rocker bogie se resalta principalmente los 2 tipos de movimiento, los cuales son el movimiento de desplazamiento plano y el movimiento rotacional para la superación de obstáculos.

La plataforma posee dos tipos de movimientos generales básicos, para su desplazamiento los cuales son el movimiento lineal, en el cual puede llegar a alcanzar velocidades de hasta $1.256 \mathrm{~km} / \mathrm{h}$ y los movimientos rotacionales que pueden ser totales o parciales.

Desplazamiento en lugares de difícil acceso. La plataforma se caracteriza por el poder desplazarse por terrenos en los cuales la dificultad de desplazamiento es alta, como lo pueden ser escaleras, las cuales puede superar gracias al sistema de amortiguamiento Roker bogie modificada, también tuvo la potencia para superar pendientes.

En el proceso de diseño y manufactura del robot se puede apreciar la importancia de la utilización de plataformas robóticas como soporte de las diferentes actividades del ser humano, ya que le brinda una reducción de riesgos a los cuales se debe exponer el individuo. Además de brindar una solución que permite ser portada a diferentes ambientes en los cuales se puede desenvolver de la mejor manera.

Teniendo en cuenta el gran impacto que puede llegar a tener el robot se desean realizar algunos cambios que contribuyan a la disminución de consumo energético para incrementar el tiempo de autonomía de la plataforma, además de realizar pruebas en ambientes extremos que la exijan al máximo y con esto observar las fallas que puedan presentarse en situaciones muy similares a las que se exponga. 


\section{Conclusiones}

El sistema mecánico implementado con una suspensión Rocker bogie modificada, permite superar las diferentes adversidades del terreno. Además de brindar gran información para la toma de decisiones como lo son imagen normal del entorno, detección de vida y temperatura.

Esta información junto con el control remoto del usuario amplia decisiones que se pueden tomar frente a una emergencia.

El sistema Epicicloide con oruga aumenta la tracción y estabilidad del robot, ya que la oruga está en contacto permanente con la superficie. Sin embargo requiere de una mayor potencia en los motores delanteros para que el sistema de rueda triangular mueva el robot con facilidad.

Se resalta que el sistema implementado disminuye el número de motores de la configuración Rocker bogie tradicional en 2 motores, haciendo un control más efectivo de la potencia que necesita el sistema en cada instante de tiempo.

\section{Recomendaciones}

Los investigadores interesados en continuar nuestra investigación podrían concentrarse en los la mejora de la circuitos y métodos de programación y en la estructura Rocker bogie.

Agradecimientos. Agradecemos las facilidades otorgadas para la realización de este trabajo al Instituto Politécnico Nacional a través de la Secretaría de Investigación y Posgrado con el proyecto SIP 20180023. A la Unidad Interdisciplinaria de Ingeniería y Ciencias Sociales y Administrativas y Centro de Investigación y Desarrollo de Tecnología Digital. Asimismo, al Programa de Estímulo al Desempeño de los Investigadores (EDI) y al Programa de Estímulo al Desempeño Docente (EDD).

\section{Referencias}

1. Montalvo Arrieta, J.C.: El potencial de riesgo sísmico en el noreste de México y sus implicaciones en la zona metropolitana de Monterrey, N. L. Ciencia UANL, Vol. XII, Núm. 4, octubre-diciembre, pp. 398-408 (2009)

2. Young, E.M., Withrow, T.J., Sarkar, N.: Design of intention-based assistive robot for upper limb. Advanced Robotics, 31(11):580-594, DOI: 10.1080/01691864.2017.1295883 (2017)

3. Chang, P., Kang, Y., Cho, G. R., Kim, J. H., Jin, M., Lee, J., Jeong, J. W., Han, D. K., Jung, J. H., Lee, W., Kim, Y.: Control Architecture Design for a Fire Searching Robot using Task Oriented Design Methodology. In: SICE-ICASE Int. Jt. Conf. 2006, pp. 31263131 (2006)

4. Kim, Young-Duk, Kim, Yoon-Gu, Lee, Seung-Hyun, Kang, Jeong-Ho, An, Jinung: Portable fire evacuation guide robot system. In: Intelligent Robots and Systems (IROS 2009), IEEE/RSJ International Conference on, pp. 2789-2794 (2009).

5. Kim, Jong-Hwan, Keller, B., Lattimer, B.Y.: Sensor fusion based seek-and-find fire algorithm for intelligent firefighting robot. In: Advanced Intelligent Mechatronics (AIM) 2013 IEEE/ASME, International Conference on, pp. 1482-1486 (2013) 
6. Amano, H.: Present status and problems of reghting robots. In: Proc. 41st SICE Annu. Conf. SICE 2002, vol. 2, pp. 880-885 (2002)

7. Harrington, B. D., Voorhees, C.: The Challenges of Designing the Rocker-Bogie Suspension for the Mars Exploration Rover. In: Proc. 37th Aerosp. Mech. Symp., pp. 185195 (2004)

8. Stauer, C., Grimson, W. E. L.: Adaptive background mixture models for real-time tracking. In: Proc. 1999 IEEE Comput. Soc. Conf. Comput. Vis. Pattern Recognit. Cat No PR00149, vol. 2, no. c, pp. 246-252 (1999)

9. Nghiem, Anh-Tuan, Bremond, F.: Background subtraction in people detection framework for RGB-D cameras. In: Advanced Video and Signal Based Surveillance (AVSS) 2014 11th IEEE International Conference on, pp. 241-246 (2014)

10. Renno, J.-P.R., Orwell, J., Jones, G.A.: Evaluation of shadow classification techniques for object detection and tracking. In: Image Processing (ICIP '04), International Conference on, vol. 1, pp. 143-146 (2004).

11. Li, Jinping, Guo, Yukun, Ma, Liwen: MCSHM: A simple and practical method for moving objects detection in dynamic scenes. In: Chinese Automation Congress (CAC), pp. 51125118 (2017) 